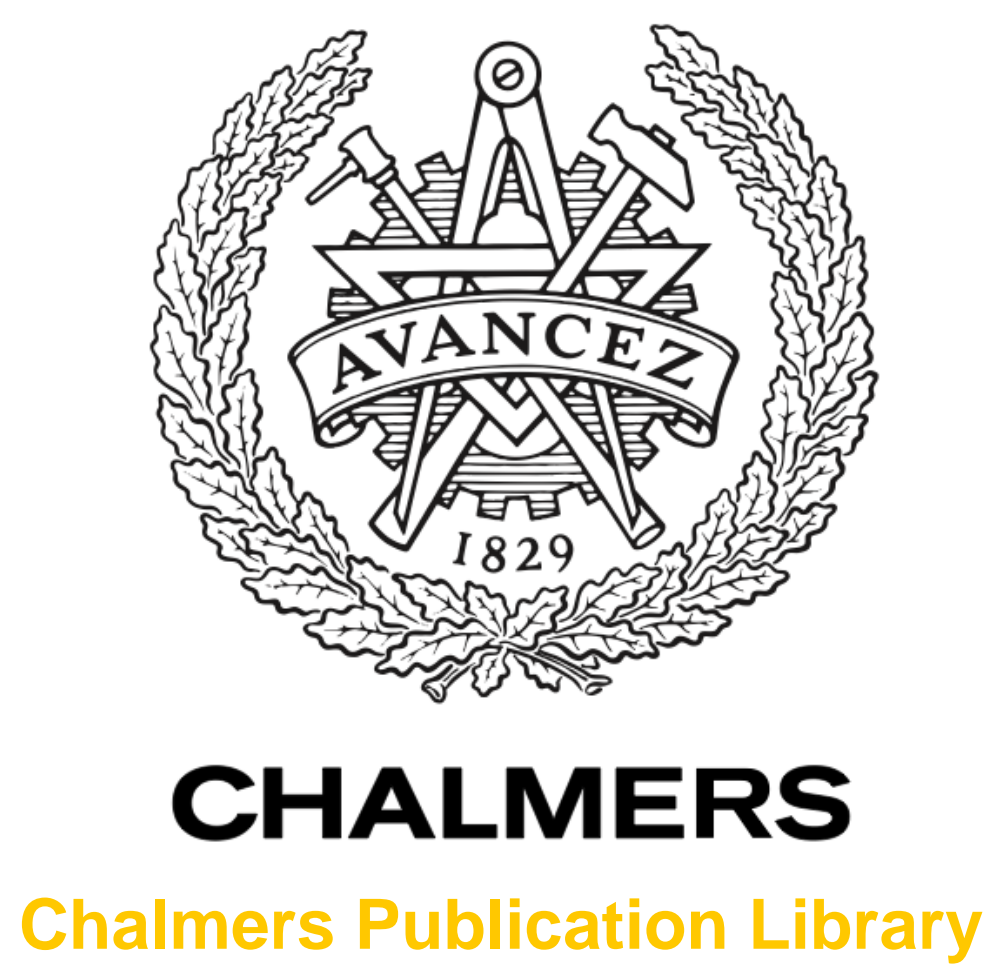

Symbol-by-Symbol Joint Polarization and Phase Tracking in Coherent Receivers

This document has been downloaded from Chalmers Publication Library (CPL). It is the author's version of a work that was accepted for publication in:

Optical Fiber Communication Conference and Exposition (OFC)

Citation for the published paper:

Czegledi, C. ; Agrell, E. ; Karlsson, M. (2015) "Symbol-by-Symbol Joint Polarization and Phase Tracking in Coherent Receivers". Optical Fiber Communication Conference and

Exposition (OFC)

Downloaded from: http://publications.lib.chalmers.se/publication/208185

Notice: Changes introduced as a result of publishing processes such as copy-editing and formatting may not be reflected in this document. For a definitive version of this work, please refer to the published source. Please note that access to the published version might require a subscription. 


\title{
Symbol-by-Symbol Joint Polarization and Phase Tracking in Coherent Receivers
}

\author{
Cristian B. Czegledi ${ }^{\S}$, Erik Agrell ${ }^{\S}$, and Magnus Karlsson \\ ${ }^{\S}$ Department of Signals and Systems, ${ }^{\ddagger}$ Department of Microtechnology and Nanoscience, \\ Chalmers University of Technology, Gothenburg, SE-412 96, Sweden. \\ czegledi@chalmers.se
}

\begin{abstract}
An analytical model to describe the combined drift of the state of polarization and absolute phase is presented. To compensate for this drift, a novel, modulation format independent algorithm is proposed, which outperforms state-of-the-art algorithms.
\end{abstract}

OCIS codes: $060.1660,060.5060$.

\section{Introduction}

Facilitated by digital signal processing (DSP), coherent detection enables spectrally efficient communication based on quadrature amplitude modulation (QAM) formats, which carry the information in both the intensity and phase of the optical field, in both polarizations. Polarization-multiplexed quadrature phase-shift keying (PM-QPSK) has been recently introduced for $100 \mathrm{~Gb} / \mathrm{s}$ transmission and it is expected that in the near future, higher-order modulation formats such as PM $M$-ary QAM (PM- $M$-QAM) will become a necessity for higher data rates. However, the improved spectral efficiency comes at the cost of a reduced tolerance to impairments such as laser phase noise and rotations of the state of polarization (SOP), which have to be dynamically tracked in the receiver [1].

Transmission of coherent data without polarization-dependent losses is typically modeled by a complex unitary $2 \times 2$ Jones transfer matrix. In [2], the Jones calculus was generalized to the four-dimensional (4-D) Euclidean space, where the evolution of the absolute phase and SOP is jointly described by a $4 \times 4$ rotation matrix with four degrees of freedom. Based on this, we propose a new method to simulate the dynamic changes of the absolute phase and SOP by modeling the four degrees of freedom as independent Wiener processes.

The common DSP solution for SOP tracking is done in the Jones space using the constant modulus algorithm (CMA) [1] or modified versions of it, such as the multiple modulus algorithm (MMA) [3] or the polarization-switched (PS-)CMA [4], which accommodate for various modulation formats. In contrast, the phase tracking is typically performed in the Euclidean space independently of the SOP tracking, using algorithms such as the Viterbi\&Viterbi algorithm [5] or the blind phase-search algorithm (BPS) [6]. The Kabsch algorithm [7] addresses the two impairments jointly over a fixed-length block of symbols and it is modulation format independent. Algorithms such as Kabsch are preferred for future, elastic optical networks, where the modulation format can be changed instantaneously to fulfill different requirements.

In this article, we propose a new system model to emulate random SOP rotations and phase noise. More importantly, to mitigate these impairments, we present a novel algorithm, which recovers the carrier phase and SOP jointly in the 4-D Euclidean space for an arbitrary constellation in a symbol-by-symbol fashion. The performance of the algorithm is investigated by comparing it with state-of-the-art algorithms for PS-QPSK, PM-QPSK, and PM-16-QAM, considering amplified spontaneous emission (ASE) noise, laser phase noise, and dynamic random SOP rotations.

\section{System Model}

The 4-D coherent optical signal can be represented by a 4-D vector and its propagation by a $4 \times 4$ real-valued rotation matrix [2]. In general, 4-D rotation matrices have six degrees of freedom, while only four of them are physically possible for propagating photons [2]. Such a rotation can be modeled with the matrix exponential of a linear combination of four basis matrices [2, Tab. I]

$$
\mathbf{R}_{k}=\exp \left(-\alpha_{1}^{(k)} \mathbf{B}_{1}-\alpha_{2}^{(k)} \mathbf{B}_{2}-\alpha_{3}^{(k)} \mathbf{B}_{3}-\alpha_{4}^{(k)} \mathbf{B}_{4}\right),
$$

where $\alpha_{i}^{(k)}$ for $i \in\{1,2,3,4\}$ are random angles at time instance $k$ and $\mathbf{B}_{i}$ are constant $4 \times 4$ basis matrices (denoted $\rho_{1,2,3}$ and $\lambda_{1}$ in [2]). The angles $\alpha_{1}^{(k)}, \alpha_{2}^{(k)}$, and $\alpha_{3}^{(k)}$ model rotations of the SOP due to birefringence and $\alpha_{4}^{(k)}$ models the phase noise. ${ }^{1}$ The typical polarization drift time can vary from microseconds up to seconds, depending on the link type and installation details. It is usually much longer than the phase drift, which is in the microsecond range for modern coherent systems [2, Tab. III].

\footnotetext{
${ }^{1}$ The relative phase retardation between the two polarizations is modeled by $\alpha_{1}^{(k)}$.
} 

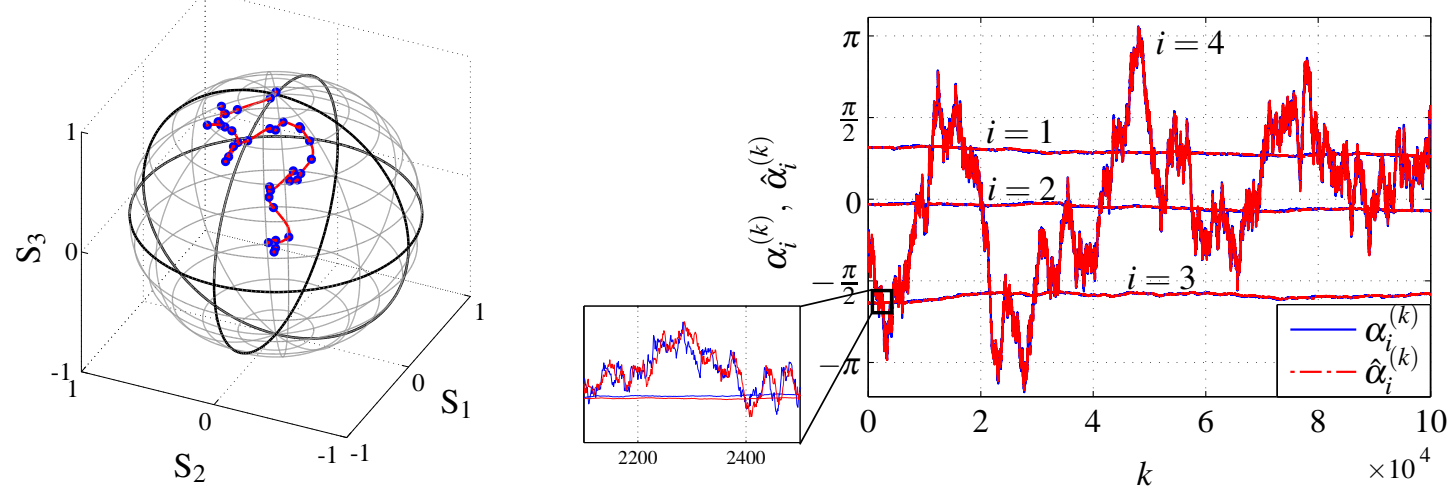

Fig. 1: Random SOP drift at $\Delta p=1 \mathrm{kHz}$. The blue dots represent samples taken at every $T=0.25 \mu \mathrm{s}$.

Fig. 2: Tracked rotation angles using the proposed algorithm at $\Delta f=2 \mathrm{MHz}$ and $\Delta p=1 \mathrm{kHz}$ for 28 Gbaud PM-QPSK.

The phase noise is usually modeled as a Wiener process [6]

$$
\alpha_{4}^{(k+1)}=\alpha_{4}^{(k)}+\delta_{f},
$$

where $\delta_{f}$ is the step size of the process, which is independently drawn at each instance $k$ from a Gaussian distribution with zero mean and variance $2 \pi \Delta f T$. Here $\Delta f$ is the cumulated linewidth of the transmitter and receiver lasers and $T$ is the symbol time. To model the SOP drift, we propose the same approach as for the phase noise, i.e., we model each angle $\alpha_{1}^{(k)}, \alpha_{2}^{(k)}$, and $\alpha_{3}^{(k)}$ as an independent Wiener process with a step size $\delta_{p, i}$, which are three independent random Gaussian variables with zero mean and variance $2 \pi \Delta p T$. We refer to $\Delta p$ as the polarization linewidth.

The received 4-D symbol $\mathbf{y}_{k}$ can be expressed as

$$
\mathbf{y}_{k}=\mathbf{R}_{k} \cdot \mathbf{x}_{k}+\mathbf{n}_{k},
$$

where $\mathbf{x}_{k}$ is the transmitted symbol and $\mathbf{n}_{k}$ is the ASE noise. An example of a random SOP drift realized with the proposed model is shown in Fig. 1, where the Stokes vector for a drifting SOP starting at $(0,0,1)$ with $T \Delta p=0.25 \cdot 10^{-3}$ are plotted on the Poincaré sphere at each $T$. Another example of a random SOP and absolute phase drift is shown with blue lines in Fig. 2.

\section{Proposed Algorithm}

The considered DSP setup is shown in Fig. 3(a), where we combine the polarization and carrier phase tracking. In order to recover the rotation in (3), we propose a blind algorithm (Fig. 3(b)) based on the stochastic gradient descent method, to minimize the error function $e_{k}=\left\|\hat{\mathbf{R}}_{k}^{-1} \cdot \mathbf{y}_{k}-\hat{\mathbf{x}}_{k}\right\|$, where $\hat{\mathbf{R}}_{k}^{-1}$ and $\hat{\mathbf{x}}_{k}$ are estimates of $\mathbf{R}_{k}$ and $\mathbf{x}_{k}$, respectively. The algorithm minimizes the error function $e_{k}$ by updating the parameters of $\hat{\mathbf{R}}_{k}$, denoted by $\hat{\alpha}_{i}^{(k)}$ in analogy with (1), as

$$
\hat{\alpha}_{i}^{(k+1)}=\hat{\alpha}_{i}^{(k)}-\mu_{i} \frac{\partial e_{k}}{\partial \hat{\alpha}_{i}^{(k)}}
$$

where $\mu_{i}$ is a positive tracking step size.

Since the typical drift time of the angles modeling the SOP rotation is slower $(\sim 1 \mathrm{~ms})$ than the angle modeling the phase noise $(\sim 1 \mu \mathrm{s})$, the tracking steps must be chosen differently. Fig. 2 shows an example of tracked rotation angles where we set $\mu_{4}=0.017$ and $\mu_{i}=4 \cdot 10^{-4}$ for $i \in\{1,2,3\}$. It can be noted that the proposed algorithm does not exhibit cycle slips over the $10^{5}$ simulated symbols.

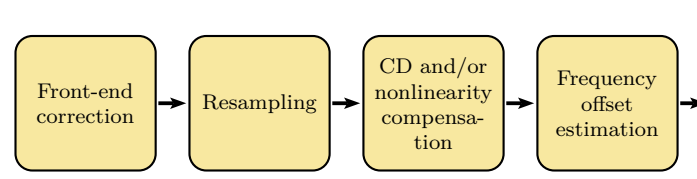

(a)

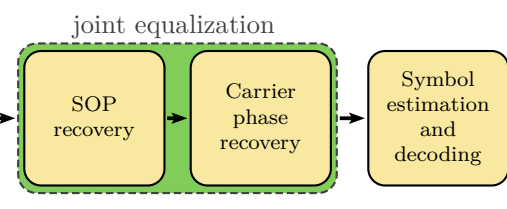

Fig. 3: (a) Receiver DSP configuration. (b) Proposed algorithm for polarization and carrier phase recovery, where $\mathbf{R}_{k}$ is estimated using (4). 


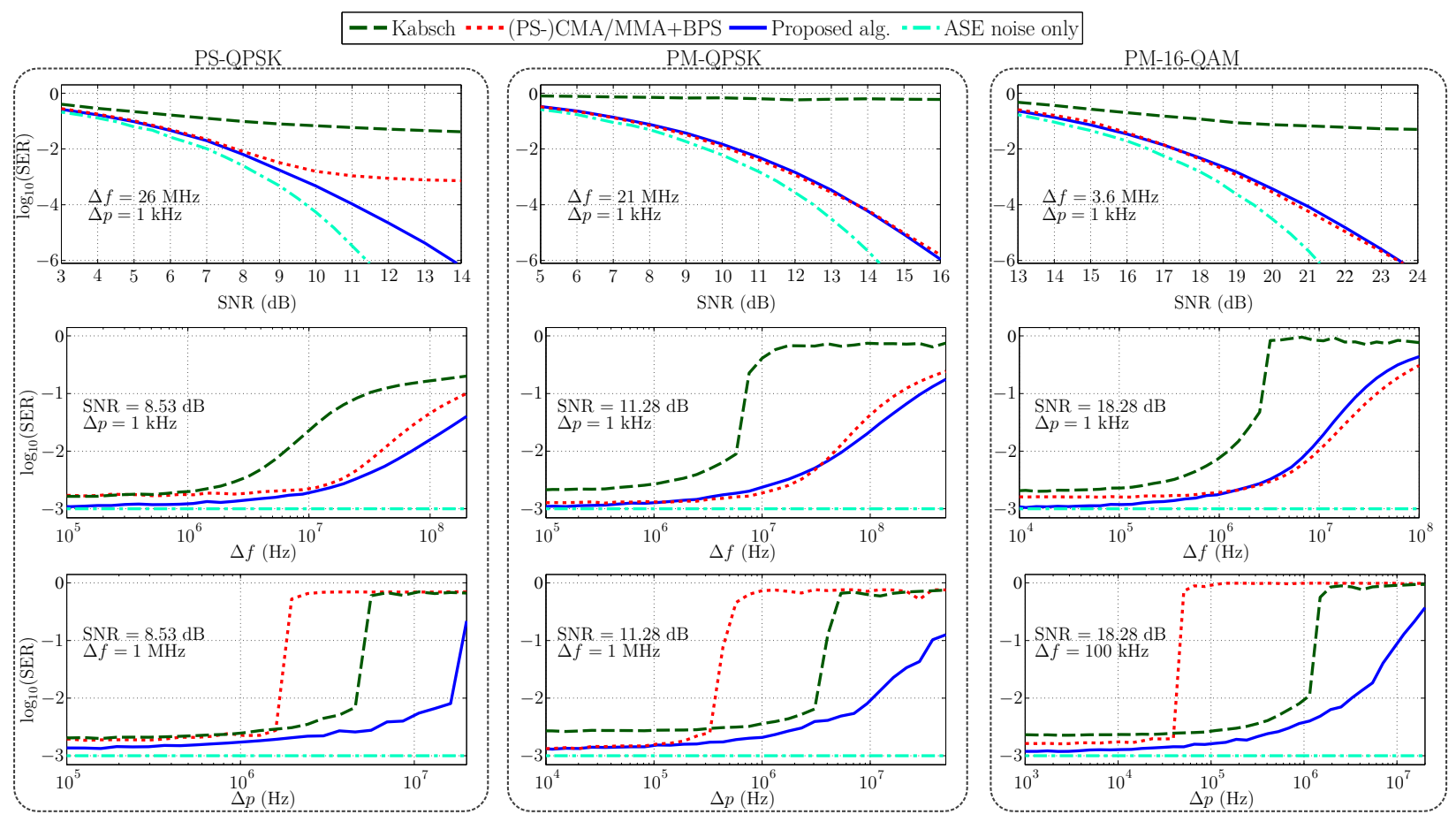

Fig. 4: Results obtained at 28 Gbaud for PS-QPSK (left), PM-QPSK (middle), and PM-16-QAM (right) showing the receiver sensitivity (top), laser phase noise tolerance (middle), and tolerance to SOP rotations (bottom).

\section{Performance Analysis}

The performance of the proposed algorithm is evaluated by means of Monte Carlo simulations in the steady-state regime in the presence of ASE noise, laser phase noise, and SOP drift. The symbol rate was set to 28 Gbaud and each data point was obtained by simulating up to $200 \cdot 10^{6}$ symbols. The tracking step size $\mu_{i}$ was chosen based on a heuristically obtained relation $\mu_{i}=\sqrt{\Delta l T c}$, where $\Delta l=\{\Delta f, \Delta p\}$ and $c$ is a constant which depends on the modulation format. The considered modulation formats are PS-QPSK ( $c=20 / 3)$, PM-QPSK $(c=4)$, and PM-16-QAM $(c=1)$, where each polarization was independently differentially encoded/decoded. For comparison, results obtained by the Kabsch algorithm [7] and combinations of the (PS-)CMA/MMA [1,3,4] and BPS [6] algorithms are presented.

Fig. 4 shows the symbol error rate (SER) as a function of: signal-to-noise ratio (SNR) at $\Delta p=1 \mathrm{kHz}$ and maximum tolerable $\Delta f$ by the proposed algorithm for a $1 \mathrm{~dB}$ SNR penalty at SER $=10^{-3}$ compared to ASE noise only (top); $\Delta f$ at $\Delta p=1 \mathrm{kHz}$ (middle); and $\Delta p$ at selected values of $\Delta f$ (bottom). In the last two cases, the SNR was set to attain SER $=10^{-3}$ in the presence of only ASE noise.

The proposed algorithm performs better than the Kabsch algorithm, and similar or better than combinations of (PS-)CMA/MMA and BPS. It is worth noting that the proposed algorithm does not suffer from the singularity problem $[1,4]$.

\section{Conclusions}

We proposed a method to emulate random SOP rotations and phase noise based on 4-D rotations. In order to mitigate these impairments in the receiver, we proposed a new, singularity-free DSP algorithm that performs joint tracking in a symbol-by-symbol fashion for any modulation format. Results show that the proposed algorithm is more tolerant to laser phase noise and SOP rotations in presence of ASE noise, which makes it a favorable choice for future receivers.

\section{References}

1. S. J. Savory, "Digital Coherent Optical Receivers: Algorithms and Subsystems," IEEE J. Sel. Topics Quantum Electron. 5, 1164-1179 (2010).

2. M. Karlsson, "Four-dimensional Rotations in Coherent Optical Communications," J. Lightw. Technol. 6, 1246-1257 (2014).

3. H. Louchet et al., "Improved DSP algorithms for coherent 16QAM transmission," European Conf. on Optical Communications, p. Tu.1.E.6 (2008).

4. P. Johannisson et al., "Modified constant modulus algorithm for polarization-switched QPSK," Opt. Express 19, 7734-7741 (2011).

5. A. J. Viterbi and A. M. Viterbi, "Nonlinear Estimation of PSKModulated Carrier Phase with Application to Burst Digital Transmission,” IEEE Trans. Inf. Theory 4, 543-551 (1983).

6. T. Pfau et al., "Hardware-Efficient Coherent Digital Receiver Concept With Feedforward Carrier Recovery for $M$-QAM Constellations," J. Lightw. Technol. 8, 989-999 (2009).

7. H. Louchet et al., "Joint Carrier-Phase and Polarization Rotation Recovery for Arbitrary Signal Constellations," IEEE Photon. Technol. Lett. 9, 922-924 (2014). 